\title{
Active Knee Orthoses - Technical Considerations and Applications
}

\author{
R. Müller ${ }^{1}$, P. Pott ${ }^{1}$, H.F. Schlaak ${ }^{1}$
}

${ }^{1}$ Microtechnology and Electromechanical Systems Lab., Institute for EMK, TU Darmstadt, Darmstadt, Germany

\section{Introduction}

Active orthoses are medical devices for active support and joint stabilization during critical and challenging situations. Currently, there is a variety of different approaches aiming to ensure this support. The objective of this work is the assessment and classification of various orthotic concepts.

\section{Assessment of orthotic concepts}

About thirty active lower limb exoskeletons are considered during a literature research. A variety of these exoskeletons also focuses on the assistance of joints like hip and/or ankle. Only seven orthotic concepts pursue the objective of sole knee support. That category is classified and assessed.

Most of the considered knee orthoses are developed for medical purposes and assistance during different gait phases. None of the orthoses are explicitly marked for the assistance during sit to stand movements (STS) that would result in increasing demands for the actuation system. In most cases, DC-motors and various kinds of gears were used to provide the desired support. Furthermore, angle and force sensors are often used to detect the orientation of lower limbs.

\section{Classification of active knee orthoses}

Basically, there are two categories of classification characteristics: technical and non-technical. The latter describes information about the potential target group as well as the magnitude and focus of supported movements. This category enables the distinction between orthotic concepts for military or medical application. The technical category describes the approach to detect the user's movement intention, the actuation concept and control concept as well as the power supply. A further aspect is the utilization of adapted knee kinematics in order to improve user comfort.

\section{Conclusion}

The challenges remain in the development of small, lightweight, and powerful actuation systems and power supplies as well as adapted kinematics in order to increase the acceptance of active knee orthoses. 